\title{
Gender differences in respiratory muscular movements in reading Japanese and English texts by JL1 and JEFL
}

Toshiko Isei-Jaakkola ${ }^{1}$, Keiko Ochi $^{2}$

${ }^{1}$ Department of English Language and Culture, Chubu University, Japan

${ }^{2}$ School of Media Science, Tokyo University of Technology, Japan

https://doi.org/10.36505/ExLing-2018/09/0013/000346

\begin{abstract}
We conducted physiological experiments to examine the gender differences in $(\mathrm{A})$ the respiratory muscles used, (B) stories read, and (C) language. For this purpose, we used respiratory strain-gauge transducers to measure chest and abdominal respiratory muscle movements and two short stories in Japanese (JL1) and English (JEFL), which were read by Japanese female and male subjects. We clarified that (1) there was a gender difference in the controlling respiratory muscles; (2) the Japanese males used the upper and lower chest muscles, and upper abdominal muscles more than did the females; (3) the language difference was not significant; and (4) the story difference was feasible.

Key words: Gender difference, Japanese speakers, Reading, English
\end{abstract}

\section{Introduction}

Abdominal and chest respirations in speech are common states. We hypothesized that females tend to use chest respiration. With regard to biological aspects in speech, Williams (1995) claimed that speech mechanisms are essentially linked to expiratory mechanisms. However, in speech production, expiration does not occur without inspiration. Saida (2015) suggested that respiratory muscular movements depend on inspiration and expiration in speech, respectively, but are inseparable.

Thus, we examined whether females use more respiratory chest muscles than respiratory abdominal muscles during reading texts than males by conducting physiological experiments, and particularly clarify the gender differences in (A) respiratory muscles used, (B) stories read, and $(\mathrm{C})$ languages. For this purpose, we used the respiratory strain-gauge transducers (RST) to measure chest and abdominal respiratory muscle movements and provided two kinds of Japanese texts (short stories: fables) in Japanese and English, which were read by the participants.

\section{Methodology}

The reading materials consisted of fables, namely "The North Wind and the Sun" (= NW) and "Momotaro" ("A Peach Boy") in Japanese and

ExLing 2018: Proceedings of 9th Tutorial and Research Workshop on Experimental Linguistics, 28-30 August, Paris, Frannce 
English. Five male and five female university students (20-25 years old) who majored in English read these two short stories five times both in Japanese and English, wearing four thin-wired RST on the upper (Chest1) and lower chests (Chest2), and the upper (Abdomen1) and lower abdomens (Abdomen2) in the sitting position (see for the detail in Isei-Jaakkola, et al., 2018). The sampling rates of the RST and speech signals were $100 \mathrm{~Hz}$ and $40 \mathrm{kHz}$, respectively. Then, we calculated six kinds of cross correlation (CC) between the following:

(a) CC1: Chest1 vs. Chest2

(b) CC2: Chest1 vs. Abdomen1

(c) CC3: Chest1 vs. Abdomen2

(d) CC4: Chest2 vs. Abdomen1

(e) CC5: Chest2 vs. Abdomen2

(f) CC6: Abdomen1 vs. Abdomen2.

The values were then used to measure the similarity of two paired muscular movements from 800-trial (3,200 signal) data by using the following procedures: In the first step, we normalized the signal waveforms by setting the mean values to 0 and the standard deviations to 1 . In the second step, we smoothed the detrended signals by using the 10-point moving average filters. Thereafter, we calculated the cross correlation between two signals at lag 0 . To measure the similarity of each pair of the RST signals, we conducted three-way analysis of variance (ANOVA; gender: male and female; languages: English and Japanese; texts: NW and, "A Peach Boy").

\section{Results}

Figure 1 shows the mean cross correlations between each pair of RST signals. The result of the three-way ANOVA revealed the significant main effects on gender in the pairs of CC1, CC2, CC4, and CC5 $(p<$ $0.05)$, but no significant interactions between gender, language, and texts. The cross correlations of CC1 and CC4, which are adjacent pairs of RST, were higher in the males than in the females. The cross correlations of CC2 and CC5, which were non-adjacent pairs, were slightly higher in the females than in the males. The result of the three-way ANOVA of the cross correlation of CC6 showed no significant main effects but showed significant interactions between gender and language, and among gender, language, and text. Post hoc analyses revealed a significant difference between genders only in the Japanese "A Peach Boy". Figure 2 shows 
the RST and speech signals when a male and a female subject read NW in English. The peaks of the Chest 1 and Chest 2 signals co-occurred in the male speaker, whereas Chest 2 of the female speaker had noticeable peaks that were not observed in Chest1.

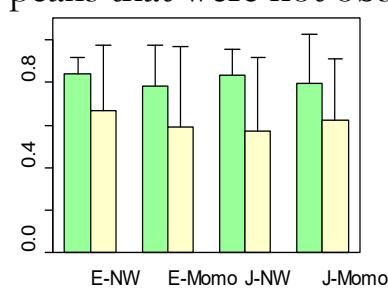

(1)Chest1 and Chest2

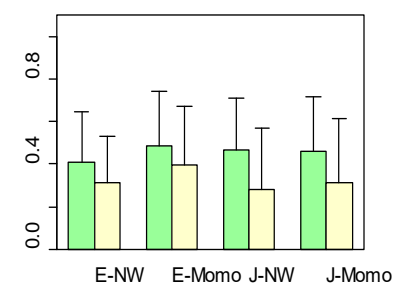

(4)Chest2 and Abdom

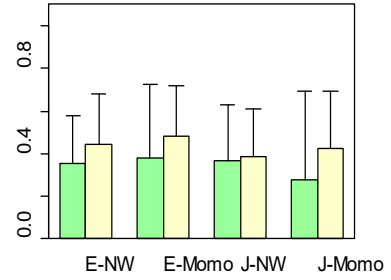

(2)Chest1 and Abdome

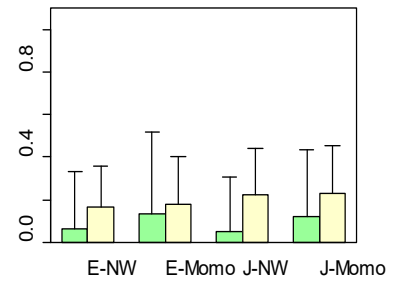

(5)Chest2 and Abdome
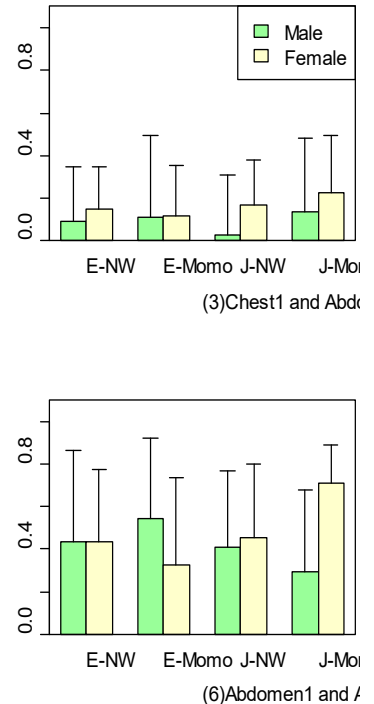

Figure 1. Six kinds of cross correlation between RST signals by the male and female subjects in English (E) and Japanese (J) according to the story (NW and Momo ("A Peach Boy"). The error bars indicate the standard deviations.
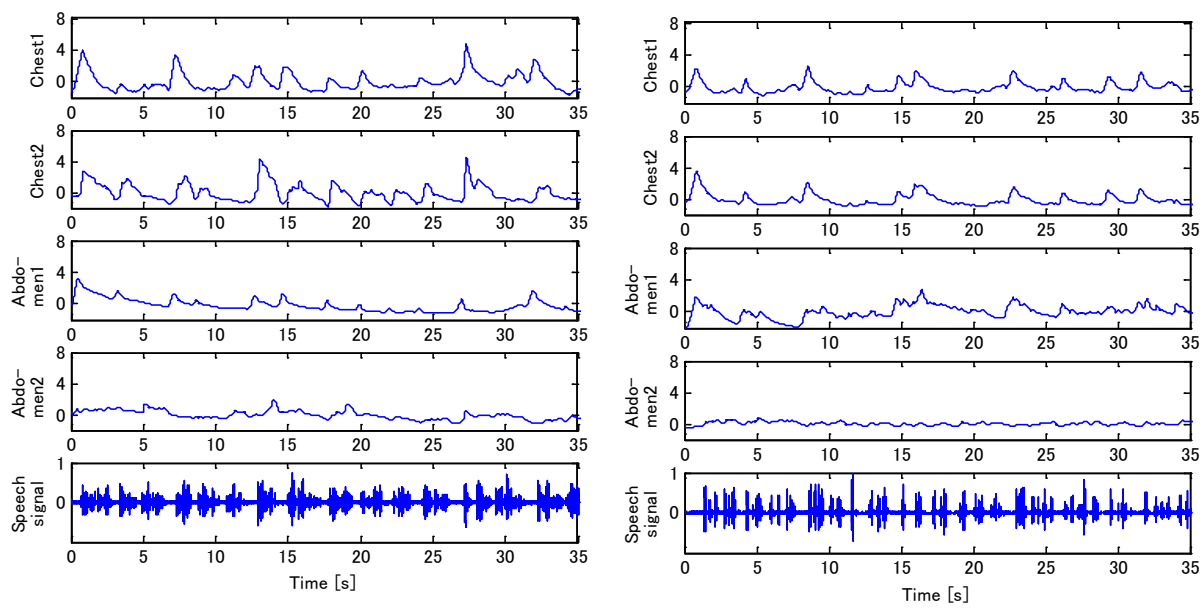

Figure 2. The RST and speech signals when a male subject (left) and a female subject (right) read NW in English. 
These results indicate that the upper and lower chest muscles were more coordinated than the upper and lower abdominal muscles for the Japanese speakers, so were those for the male subjects than for the female subjects. On the other hand, the upper and lower chest and lower abdomen were not coordinated.

\section{Conclusions}

We clarified that (1) a gender difference in the controlling respiratory muscles existed as far as the Japanese speakers were concerned under these experimental conditions and that they control respiratory muscles according to language and story, although the significance was not necessarily high. The Japanese males used upper and lower chest muscles and upper abdominal muscles more than did the females during reading. Both the upper and lower chest were highly coordinated but the lower abdomen was not coordinated with the chest and upper abdominal muscles. This implies that the upper and lower chest, and the upper abdomen are biologically inevitably more linked with respiration during speech, but the lower abdominal muscle could be controlled by more arbitrary efforts (cf., Isei-Jaakkola, 2015). (2) In addition, the difference between stories affected only the females. (3) Furthermore, no significant difference in language was observed. This may prove that JEFL used the respiratory muscle movements of JL1 in reading a targeted language to learn. Our hypothesis must be further investigated with other language speakers to reconfirm our present results.

\section{Acknowledgements}

This study is partly supported by Grant-in-Aid KAKENHI for Scientific Research C (NO. 17K02698) of Japan.

\section{References}

Isei-Jaakkola, T, Ochi, K., Hirose, K. 2018. Respiratory and Respiratory Muscular in JL1's and JL2's Text Reading Utilizing 4-RSTs and a Soft Respiratory Mask with a Two-Way Bulb. Proc. of INTERSPEECH 2018. (Accepted).

Saida, H. 2016. Medical Voice Designer Assists You In Your Vocalization Using Newly Developed Voice Maps (in Japanese). Ongaku No Tomo Sha.

Williams, P. L. 1995. Gray's Anatomy: The Anatomical Basis of Medicine and Surgery, 38e (Gray's Anatomy: the Anatomical Basis of Clinical Practice), Churchhill Livingstone. 